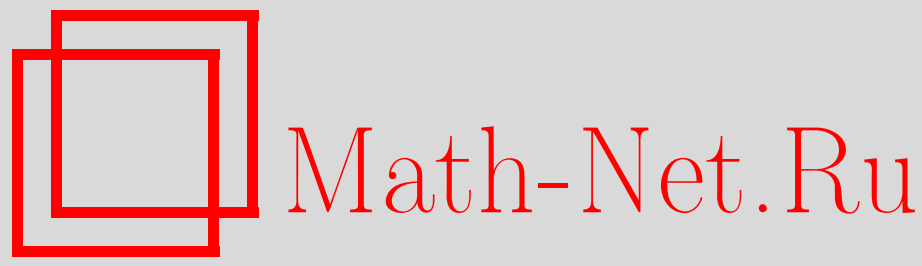

Я. В. Татаринов, Формализм релятивистской динамики нескольких материальных точек и прецессия орбиты в задаче "шар-точка", ТMФ, 2011, том 166, номер 3, 425442

DOI: https://doi.org/10.4213/tmf6620

Использование Общероссийского математического портала Math-Net.Ru подразумевает, что вы прочитали и согласны с пользовательским соглашением http: //www.mathnet.ru/rus/agreement

Параметры загрузки:

IP : 18.208 .226 .222

26 апреля 2023 г., 17:09:15

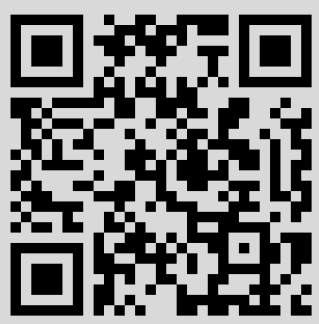




\title{
ФОРМАЛИЗМ РЕЛЯТИВИСТСКОЙ ДИНАМИКИ НЕСКОЛЬКИХ МАТЕРИАЛЬНЫХ ТОЧЕК И ПРЕЦЕССИЯ ОРБИТЫ В ЗАДАЧЕ "ШАР-ТОЧКА"
}

\begin{abstract}
В рамках подхода Козлова и Никишина рассматривается релятивистская задача о динамике гравитирующих шара и точки. Используется лагранжев формализм. Лагранжиан является инвариантным относительно группы Пуанкаре. С помощью теории возмущений установлено существование прецессии орбиты точки.
\end{abstract}

Ключевые слова: теория относительности, задача двух тел, прецессия орбиты.

\section{1. ВВЕДЕНИЕ}

Пуанкаре писал в конце XIX в. (см. монографию [1], введение): "Конечная цель небесной механики состоит в разрешении великого вопроса, может ли закон Ньютона, и только он один, объяснить все астрономические явления". Разумеется, тогда он имел в виду классическую механику, но сейчас мы имеем возможность - и в большой степени благодаря именно Пуанкаре - смотреть на вещи шире [2]. Однако та релятивистская динамика, которая есть во всех учебниках, описывает движение только одной точки, так как рассмотрение системы материальных точек сталкивается с существенной проблемой. Как пишет Синг [3], “мы имеем только множество мировых линий", и "чтобы установить соответствие между несколькими событиями и одним и тем же значением времени, не существует никакого лоренц-инвариантного метода". Предложения разрешить проблему основывались на наложении связей, например, с применением гамильтонова формализма Дирака [4] (обсуждение истории таких подходов см. в работе [5]). Есть более красивое предложение: свободная (т. е. без связей) гамильтонова динамика (СГМ) в пространстве-времени из статьи [6]. Рассматривается несколько частиц с индивидуальным координатным временем у каждой, независимая переменная не привязана ни к какой материальной частице, лагранжиан и гамильтониан лоренц-инвариантны. Сопоставление этого подхода со специальной теорией относительности (включая трактовку одновременности) проведено в работе [7].

\footnotetext{
* Московский государственный университет им. М. В. Ломоносова, Москва, Россия
} 
Лоренц-инвариантная задача двух тел в СГМ имеет много интегралов, в силу чего все ограниченные траектории периодичны. В работе [8] то же доказано иначе: введены переменные действие-угол (аналоги канонических элементов Делоне задачи Кеплера [9], в которых гамильтониан вырожден, а ограниченное движение характеризуется только одной частотой, как и в задаче Кеплера, и происходит по эллипсу).

В настоящей работе мы заменим одну из точек на тело, “твердое" относительно группы Пуанкаре, выпишем уравнения движения системы "шар-точка" и найдем, когда вращение шара отделяется от орбитального движения; затем применим теорию возмущений [10] на основе решения задачи двух тел из работ [6], [7]. Появляется прецессия бывшей эллиптической орбиты.

Используются стандартные сведения о псевдоевклидовых пространствах [11]-[14], однако будут опущены почти все ссылки на эти книги, равно как и на статьи [6], [8]. При выводе уравнений движения используется их новая форма [15], [16]. Развитие квантово-механических аспектов статьи [6] можно найти в работах [17]-[20].

\section{2. ЛАГРАНЖИАН И ГАМИЛЬТОНИАН}

Рассмотрим пространство $\{(x, y, z, t)\}$ с псевдоевклидовой метрикой $d x^{2}+d y^{2}+$ $d z^{2}-c^{2} d t^{2}$ и (псевдо)скалярным произведением, которое обозначим $\langle\cdot, \cdot\rangle$. Чуть позже мы применим и тензорные обозначения $\left(x^{1}, x^{2}, x^{3}\right)=(x, y, z), x^{4}=c t$. Допуская вольность, согласимся писать

$$
\mathbf{q}=(\mathbf{r}, t)=(x, y, z, t)=\left(x^{1}, x^{2}, x^{3}, x^{4}\right), \quad \boldsymbol{\sigma}=(\xi, \eta, \zeta, \tau)=\left(\xi^{1}, \xi^{2}, \xi^{3}, \xi^{4}\right)
$$

Сюда можно приписывать нижние индексы. Станем рассматривать движение нескольких частиц с координатами $\left(x_{\nu}, y_{\nu}, z_{\nu}, t_{\nu}\right)$. Роль независимого переменного играет величина $s$, имеющая размерность времени. Это совсем не координатное время, наблюдаемое в данной системе отсчета. В координатном времени ньютоново понимание выражения "сейчас" сохраняет смысл (так или иначе) применительно если не ко всему пространству, то хотя бы в некоторой области вокруг начала координат. В СГМ вводится свое понимание одновременности [7]: это все события при заданном значении $s$. Здесь перед нами встает проблема физической, операционной интерпретации развиваемого формализма (как и любого другого): какими способами измеряются координата $t_{i}$ для каждой точки и “всеобщая" координата $s$, какую систему отсчета - на каких основаниях, с какой точностью - разумно считать инерциальной (ускорения точек обусловлены только присутствием их самих или, возможно, и иных материальных объектов (и каких, кстати?)) и т. д.

Производная по $s$ обозначается штрихом.

Фундаментальный лагранжиан. Запишем его в общем виде

$$
\begin{gathered}
\mathcal{L}=\frac{1}{2} \sum_{\nu=1}^{N} m_{\nu}\left(-c^{2} t_{\nu}^{\prime 2}+x_{\nu}^{\prime 2}+y_{\nu}^{\prime 2}+z_{\nu}^{\prime 2}\right)-V\left(\ldots, \Delta_{\nu \varkappa}, \ldots\right), \\
\Delta_{\nu \varkappa}=\sqrt{\left(x_{\nu}-x_{\varkappa}\right)^{2}+\left(y_{\nu}-y_{\varkappa}\right)^{2}+\left(z_{\nu}-z_{\varkappa}\right)^{2}-c^{2}\left(t_{\nu}-t_{\varkappa}\right)^{2}}, \quad M=\sum m_{\nu},
\end{gathered}
$$


инвариантном относительно группы Пуанкаре (синоним: лоренц-инвариантном). Он определен в следующей лоренц-инвариантной области фазового пространства:

$$
\Lambda=\left\{\frac{\left(r_{\nu}-r_{\varkappa}\right)^{2}}{c^{2}}-\left(t_{\nu}-t_{\varkappa}\right)^{2} \geqslant 0\right\} \text {. }
$$

Гамильтониан (здесь $\left.p_{r}=\left(p_{x}, p_{y}, p_{z}\right)\right)$ имеет вид

$$
\mathcal{H}=\sum \frac{1}{2 m_{\nu}}\left(p_{r_{\nu}}^{2}-\frac{1}{c^{2}} p_{t_{\nu}}^{2}\right)+V=h .
$$

Для случая гравитационного взаимодействия $V=-f \sum_{\nu<\varkappa} m_{\nu} m_{\varkappa} / \Delta_{\nu \varkappa}$.

Интеграл энергии для рассматриваемого лагранжиана записывается как

$$
\frac{1}{2} \sum m_{\nu}\left(-\left(c^{2} \frac{d t_{\nu}}{d s}\right)^{2}+\left(\frac{d r_{\nu}}{d s}\right)^{2}\right)+V=h .
$$

Вложенная классика [6]. 1. При $c \rightarrow \infty$ из формулы $(1)$ имеем $\left(t_{\nu}-t_{\varkappa}\right) \rightarrow 0$, так что $\mathcal{H}$ принимает обычный ньютонов вид. 2 . Более того, решения с $\left(t_{\nu}-t_{\varkappa}\right)=0$ образуют инвариантное многообразие классических решений в фазовом пространстве.

Таким образом, при подходящем выборе начальных условий можно прийти в точности к классическим решениям или сколь угодно близким к ним. Скажем коротко так: "классика вложена".

Выделение центра масс. Будем обозначать одной буквой значения масс и геометрические точки, в которых массы находятся. Точки с номерами 2 и выше образуют у нас "тело". Обозначим $\sum^{2}=\sum_{\nu=2}^{N}$ и согласимся считать, что $m_{2}$ есть центр масс тела, т. е.

$$
\mathbf{q}_{2}=\frac{1}{M_{2}} \sum^{2} m_{\nu} \mathbf{q}_{\nu}, \quad M_{2}=\sum^{2} m_{\nu}
$$

Это определение не зависит от выбора системы координат в отличие от приведенного в книге [14], §33.

Для точек тела обозначим $\mathbf{q}_{\nu}=\mathbf{q}_{2}+\boldsymbol{\sigma}_{\nu}$. Пусть также $S(X, Y, Z, T)$ - центр масс всей системы точек. Положим

$$
\mathbf{q}_{1}=\mathbf{q}_{S}+\frac{M_{2}}{M} \mathbf{q}, \quad \mathbf{q}_{2}=\mathbf{q}_{S}-\frac{m_{1}}{M} \mathbf{q}, \quad \mu=\frac{m_{1} M_{2}}{M} .
$$

В итоге лагранжиан принимает вид

$$
\mathcal{L}=\frac{M\left\langle\mathbf{q}_{S}^{\prime}, \mathbf{q}_{S}^{\prime}\right\rangle}{2}+\frac{\mu\left\langle\mathbf{q}^{\prime}, \mathbf{q}^{\prime}\right\rangle}{2}+\sum^{2} \frac{m_{\nu}\left\langle\boldsymbol{\sigma}_{\nu}^{\prime}, \boldsymbol{\sigma}_{\nu}^{\prime}\right\rangle}{2}-V
$$

Сразу ясно, что отделяется движение общего центра масс (оно равномерное), и в дальнейшем упоминать его мы не будем. 
Две точки. При $N=2$ "тело" сводится к точке. Динамика задается гамильтонианом

$$
\mathcal{H}=\frac{1}{2 M}\left(p_{r_{1}}^{2}-\frac{p_{t_{1}}^{2}}{c^{2}}\right)+\frac{1}{2 \mu}\left(p_{r_{2}}^{2}-\frac{p_{t_{2}}^{2}}{c^{2}}\right)+V(\rho),
$$

где $\rho-($ псевдо)расстояние между этими точками. Гамильтониан движения есть

$$
\mathcal{H}=\frac{1}{2 M}\left(c^{2} p_{R}^{2}-p_{T}^{2}\right)+H, \quad H=\frac{1}{2 \mu}\left(p_{r}^{2}-\frac{p_{t}^{2}}{c^{2}}\right)+V(\rho) .
$$

Обратим внимание, что координаты q, относимые формально к приведенной массе $\mu$, суть разности соответствующих координат точек, в частности переменная $t$ есть разность собственных времен точек и может быть даже нулем.

Гармонический осциллятор. Рассмотрим две точки и потенциальную энергию

$$
V(\rho)=K\left(\left(x_{1}-x_{2}\right)^{2}+\left(y_{1}-y_{2}\right)^{2}+\left(z_{1}-z_{2}\right)^{2}-c^{2}\left(t_{1}-t_{2}\right)^{2}\right) .
$$

Тогда после отделения движения центра масс для разностей координат получим уравнения Лагранжа

$$
m x^{\prime \prime}+K x=0, \quad m y^{\prime \prime}+K y=0, \quad m z^{\prime \prime}+K z=0, \quad-c^{2} t^{\prime \prime}-K c^{2} t=0,
$$

т. е. по всем переменным происходят колебания с одной и той же частотой. Временнь́е координаты частиц "не разбегаются". Если же взять отталкивающую силу упругости, то вывод будет противоположным. Кстати, в этой задаче ограничений на область определения потенциала нет.

Твердое тело. Будем называть тело "твердым", если $\Delta_{\nu \varkappa}=l_{\nu \varkappa}=$ const, $\nu, \varkappa \geqslant$ 2 . Кавычки поставлены потому, что интервалы $\Delta_{\nu \varkappa}$ сохраняются и при преобразованиях Лоренца, например

$$
\tilde{x}=\frac{x+u t}{\sqrt{1-u^{2} / c^{2}}}, \quad \tilde{t}=\frac{t+u x / c^{2}}{\sqrt{1-u^{2} / c^{2}}} .
$$

Кинематическая трактовка последних говорит об изменении наблюдаемых евклидовых расстояний при переходе от одной системы отсчета к другой. Поэтому чистые вращения (не затрагивающие временну́ю координату) представляют особую ценность. Они образуют подгруппу.

Для “твердого тела" третье слагаемое в лагранжиане (6) имеет смысл кинетической энергии псевдоевклидова вращения $\mathcal{T}_{\text {вращ }}$

\section{3. ОДНОПАРАМЕТРИЧЕСКИЕ ГРУППЫ (ПСЕВДО)ИЗОМЕТРИЙ}

В фазовом пространстве $\left\{\left(x, y, z, t, p_{x}, p_{y}, p_{z}, p_{t}\right)\right\}$ введем функции (компоненты бивектора кинетического момента)

$$
\begin{aligned}
& F_{1}=\Phi_{3}^{2}=y p_{z}-z p_{y}, \quad F_{2}=\Phi_{1}^{3}=z p_{x}-x p_{z}, \quad F_{3}=\Phi_{2}^{1}=x p_{y}-y p_{x}, \\
& F_{4}=\Phi_{1}^{4}=\frac{1}{c} x p_{t}+c p_{x} t, \quad F_{5}=\Phi_{2}^{4}=\frac{1}{c} y p_{t}+c t p_{y}, \quad F_{6}=\Phi_{3}^{4}=\frac{1}{c} z p_{t}+c t p_{z} .
\end{aligned}
$$


Они являются первыми интегралами движения приведенной массы и задают тензор $\Phi$, кососимметрический относительно $\langle\cdot, \cdot\rangle$. Отсутствие минусов во второй строке связано с тем, что $p_{t}=-c^{2} t^{\prime}$.

Рассмотрим векторные поля и фазовые потоки, задаваемые этими гамильтонианами:

$$
\begin{array}{ll}
\overleftarrow{F}_{1}: & g^{s}\left(\begin{array}{l}
y \\
z
\end{array}\right)=\left(\begin{array}{cc}
\cos s & -\sin s \\
\sin s & \cos s
\end{array}\right)\left(\begin{array}{l}
y \\
z
\end{array}\right) \\
\overleftarrow{F}_{4}: & g^{s}\left(\begin{array}{l}
x \\
t
\end{array}\right)=\left(\begin{array}{cc}
\operatorname{ch} s & c \operatorname{sh} s \\
\operatorname{sh} s / c & \operatorname{ch} s
\end{array}\right)\left(\begin{array}{l}
x \\
t
\end{array}\right) .
\end{array}
$$

Неупомянутые координаты не изменяются. Перед нами "чистый” поворот и стандартное преобразование Лоренца (взяв th $s=u / c$, получим (9)).

Пусть $i=1,2,3(\bmod 3)$. Попарные скобки Пуассона имеют вид

$$
\begin{array}{ll}
\left\{F_{i+1}, F_{i+2}\right\}=F_{i}, & \left\{F_{i+1}, F_{i+5}\right\}=F_{i+4}, \\
\left\{F_{i+1}, F_{i+6}\right\}=-F_{i+4}, & \left\{F_{i+5}, F_{i+6}\right\}=-F_{i} .
\end{array}
$$

Не написаны нулевые скобки и получающиеся по косой симметрии. Перечисленные функции порождают алгебру so $(3,1)$.

Запишем также попарные скалярные произведения полей:

$$
\begin{aligned}
\left\langle F_{i+1}, F_{i+2}\right\rangle & =\left\langle F_{i+4}, F_{i+5}\right\rangle=x^{i+1} x^{i+2}, & & \left\langle F_{i+1}, F_{i+5}\right\rangle=x^{i} x^{4}, \\
\left\langle F_{i}, F_{i}\right\rangle & =x^{i+1^{2}}+x^{i+2^{2}}, & & \left\langle F_{i+3}, F_{i+3}\right\rangle=x^{i^{2}} .
\end{aligned}
$$

\section{4. ПРОСТЕЙШИЕ ОСКУЛИРУЮЩИЕ ЭЛЕМЕНТЫ ЗАДАЧИ "ТОЧКА-ТОЧКА"}

Введем координаты для той части пространства $(x, y, z, t)$, где $\langle\mathbf{q}, \mathbf{q}\rangle>0$ :

$x=\rho \operatorname{ch} \psi \cos \theta \cos \varphi, \quad y=\rho \operatorname{ch} \psi \cos \theta \sin \varphi, \quad z=\rho \operatorname{ch} \psi \sin \theta, \quad c t=\rho \operatorname{sh} \psi$.

В дальнейшем потенциал имеет вид

$$
V(\rho)=-\frac{\mu G}{\rho}
$$

Будем рассматривать функцию Гамильтона расширенной задачи Кеплера:

$$
H=\frac{1}{2 \mu}\left(p_{\rho}^{2}+\frac{1}{\rho^{2}}\left(-p_{\psi}^{2}+\frac{1}{\operatorname{ch}^{2} \psi}\left(p_{\theta}^{2}+\frac{p_{\varphi}^{2}}{\cos ^{2} \theta}\right)\right)\right)-\frac{\mu G}{\rho} .
$$

Видно, что переменные $\varphi, \theta, \psi, \rho$ по очереди отделяются. Первые интегралы равны

$$
\begin{gathered}
p_{\varphi}=C_{4}, \quad p_{\theta}^{2}+\frac{C_{4}^{2}}{\cos ^{2} \theta}=C_{3}, \quad-p_{\psi}^{2}+\frac{C_{3}}{\operatorname{ch}^{2} \psi}=C_{2}, \\
H=\frac{1}{2 \mu}\left(p_{\rho}^{2}+\frac{C_{2}}{\rho^{2}}\right)-\frac{\mu G}{\rho}=C_{1}=h .
\end{gathered}
$$

Траектории существуют, ограничены и бесстолкновительны при условиях

$$
0<C_{4}\left(=\alpha_{4}^{2}\right) \leqslant C_{3}\left(=\alpha_{3}^{2}\right), \quad 0 \leqslant C_{2}\left(=\alpha_{2}^{2}\right) \leqslant \alpha_{3}^{2}, \quad 0>h\left(=-\frac{\mu^{3} G^{2}}{\alpha_{1}^{2}}\right), \quad \alpha_{1}^{2} \geqslant \alpha_{2}^{2} .
$$


ЛЕммА. В задаче с гамилътонианом (12) инвариантны подмногообразия $\psi=0$, $p_{\psi}=0$ (классическая подзадача), $\theta=0, p_{\theta}=0$ (движение в плоскости $z=0$, что не уменьшает общности, так как эту ось всегда можно направить по кинетическому моменту $\left.\left(F_{1}, F_{2}, F_{3}\right)\right)$.

Пусть ради краткости $\mu=1$. Вторая часть задачи сводится к движению единичной массы в псевдоевклидовом пространстве $\mathbb{R}^{2,1}=\left\{x^{1}, x^{2}, x^{4}\right\}$. В нем вводится аналог векторного произведения (не зависящего от выбора псевдоортонормированного репера):

$$
\begin{gathered}
{[a \wedge b]=\left|\begin{array}{ccc}
-\mathbf{e}_{1} & -\mathbf{e}_{2} & \mathbf{e}_{4} \\
a^{1} & a^{2} & a^{4} \\
b^{1} & b^{2} & b^{4}
\end{array}\right|,} \\
{\left[\mathbf{e}_{1} \wedge \mathbf{e}_{2}\right]=\mathbf{e}_{4}, \quad\left[\mathbf{e}_{4} \wedge \mathbf{e}_{1}\right]=-\mathbf{e}_{2}, \quad\left[\mathbf{e}_{4} \wedge \mathbf{e}_{2}\right]=\mathbf{e}_{1},} \\
\langle[\mathbf{a} \wedge \mathbf{b}], \mathbf{a}\rangle=0, \quad \operatorname{det}\|\mathbf{a}, \mathbf{b}, \mathbf{a} \wedge \mathbf{b}\|=-\langle[\mathbf{a} \wedge \mathbf{b}],[\mathbf{a} \wedge \mathbf{b}]\rangle .
\end{gathered}
$$

Стало быть, векторы $\mathbf{a}, \mathbf{b}, \mathbf{a} \wedge \mathbf{b}$ составляют правую тройку, когда третий из них времениподобен.

ЗАмЕчАниЕ. Непривычной чертой псевдоевклидова пространства является то, что $a^{4}=-\left\langle\mathbf{a}, \mathbf{e}_{4}\right\rangle$. Обозначим обычное векторное произведение $[a, b]$. Тогда имеем $[a \wedge b]=-D^{1}[a, b]=\left[D^{1} a, D^{1} b\right]$, где $D^{1}$ - матрица Грама базиса; наконец,

$$
[\mathbf{a} \wedge[\mathbf{b} \wedge \mathbf{c}]]=-\mathbf{b}\langle\mathbf{a}, \mathbf{c}\rangle+\mathbf{c}\langle\mathbf{a}, \mathbf{b}\rangle,
$$

откуда вытекает тождество Якоби для произведения $\langle\cdot, \cdot\rangle$.

Удобные реперы. В силу формулы (11) имеем

$$
x=\rho \operatorname{ch} \psi \cos \varphi, \quad y=\rho \operatorname{ch} \psi \sin \varphi, \quad c t=\rho \operatorname{sh} \psi .
$$

Будем использовать (псевдо)ортонормированный координатный репер:

$$
\begin{gathered}
\mathbf{f}_{1}=\left(\begin{array}{c}
\operatorname{ch} \psi \cos \varphi \\
\operatorname{ch} \psi \sin \varphi \\
\operatorname{sh} \psi
\end{array}\right), \quad \mathbf{f}_{2}=\left(\begin{array}{c}
-\sin \varphi \\
\cos \varphi \\
0
\end{array}\right), \quad \mathbf{f}_{4}=\left(\begin{array}{c}
\operatorname{sh} \psi \cos \varphi \\
\operatorname{sh} \psi \sin \varphi \\
\operatorname{ch} \psi
\end{array}\right), \\
\mathbf{q}=\rho \mathbf{f}_{1}, \quad \mathbf{q}^{\prime}=\rho^{\prime} \mathbf{f}_{1}+\rho \varphi^{\prime}(\operatorname{ch} \psi) \mathbf{f}_{2}+\rho \psi^{\prime} \mathbf{f}_{4}
\end{gathered}
$$

Следовательно, аналог кинетического момента (времениподобный вектор) имеет вид

$$
\begin{gathered}
\Lambda=\left[\mathbf{q} \wedge \mathbf{q}^{\prime}\right]=\rho^{2} \psi^{\prime} \mathbf{f}_{2}+\rho^{2} \varphi^{\prime}(\operatorname{ch} \psi) \mathbf{f}_{4} \\
\langle\boldsymbol{\Lambda}, \boldsymbol{\Lambda}\rangle=\rho^{4}\left(\psi^{\prime 2}-\left(\operatorname{ch}^{2} \psi\right) \varphi^{\prime 2}\right)=-\left(-p_{\psi}^{2}+\frac{p_{\theta}^{2}}{\operatorname{ch}^{2} \psi}\right)=-\alpha_{2}^{2} .
\end{gathered}
$$

Введем еще один (псевдо)ортонормированный репер:

$$
\begin{gathered}
\mathbf{n}_{1}=\left(\begin{array}{c}
\cos \chi \\
\sin \chi \\
0
\end{array}\right), \quad \mathbf{n}_{2}=\left(\begin{array}{c}
-\operatorname{ch} \lambda \sin \chi \\
\operatorname{ch} \lambda \cos \chi \\
\operatorname{sh} \lambda
\end{array}\right), \quad \mathbf{n}_{4}=\left(\begin{array}{c}
\operatorname{sh} \lambda \sin \chi \\
-\operatorname{sh} \lambda \cos \chi \\
\operatorname{ch} \lambda
\end{array}\right), \\
\mathbf{q}=\rho \mathbf{f}_{1}=\rho\left((\cos \nu) \mathbf{n}_{1}+(\sin \nu) \mathbf{n}_{2}\right), \\
\mathbf{q}^{\prime}=\rho^{\prime}\left((\cos \nu) \mathbf{n}_{1}+(\sin \nu) \mathbf{n}_{2}\right)+\rho \nu^{\prime}\left(-(\sin \nu) \mathbf{n}_{1}+(\cos \nu) \mathbf{n}_{2}\right) .
\end{gathered}
$$


Здесь $\lambda, \chi, \nu$ - аналоги наклона орбиты, долготы восходяшего узла и аргумента широты из задачи Кеплера (величину $i \lambda$ можно называть мнимым наклоном, так как $\cos i \lambda=\operatorname{ch} \lambda)$. Теперь

$$
\begin{gathered}
\boldsymbol{\Lambda}=\Lambda \mathbf{n}_{4}, \quad \Lambda=\rho^{2} \nu^{\prime}=\alpha_{2}, \\
p_{\varphi}=\Lambda_{4}=\Lambda \operatorname{ch} \lambda=\rho^{2}\left(\operatorname{ch}^{2} \psi\right) \varphi^{\prime}=\rho^{2} \operatorname{ch} \lambda \nu^{\prime}=\alpha_{4} .
\end{gathered}
$$

Впредь для определенности будем считать $\alpha_{4}>0$, так что $\varphi^{\prime}, \nu^{\prime}>0$.

Интегрирование. В гамильтониане (12) в силу леммы положим $\theta=0, p_{\theta}=0$ (тогда в формулах (13), (14) $C_{3}=C_{4}^{2}$ ) и для ограниченных движений напишем полный интеграл уравнения Гамильтона-Якоби в виде

$$
S\left(\alpha_{1}, \alpha_{2}, \alpha_{3}, \rho, \psi, \varphi\right)=\alpha_{3} \varphi+\int_{0}^{\psi} \sqrt{\frac{\alpha_{3}^{2}}{\operatorname{ch}^{2} \psi}-\alpha_{2}^{2}} d \psi+\int_{\rho_{\min }}^{\rho} \sqrt{-\frac{G^{2}}{\alpha_{1}^{2}}+\frac{2 G}{\rho}-\frac{\alpha_{2}^{2}}{\rho^{2}}} d \rho .
$$

Выпишем формулы перехода к новым каноническим переменным:

$\beta_{4}(\bmod 2 \pi)=\frac{\partial S}{\partial \alpha_{4}}=\varphi-\alpha_{4} \int_{0}^{\psi} \frac{d \psi}{\operatorname{ch}^{2} \psi \sqrt{\alpha_{4}^{2}-\alpha_{2}^{2} / \operatorname{ch}^{2} \psi}}=\varphi+\arcsin \left(\frac{\alpha_{4}}{\sqrt{\alpha_{4}^{2}-\alpha_{2}^{2}}} \operatorname{th} \psi\right)$, или

$$
\text { th } \lambda \sin \left(\beta_{4}-\varphi\right)=\text { th } \psi \text {. }
$$

То же самое получится, если спроектировать (17) на $\mathbf{n}_{4}$ :

$$
\operatorname{ch} \psi \operatorname{sh} \lambda \sin (\varphi-\chi)+\operatorname{sh} \psi \operatorname{ch} \lambda=0 .
$$

Отсюда $\beta_{4}=\chi$ - аналог долготы восходящего (если $\nu=0$, то $\left.x_{4}^{\prime}=\rho^{2}(\operatorname{ch} \psi) \nu^{\prime}>0\right)$ узла. Далее, положим

$$
\begin{gathered}
\frac{\alpha_{2}}{\rho}-\frac{G}{\alpha_{2}}=G \sqrt{\frac{\alpha_{1}^{2}-\alpha_{2}^{2}}{\alpha_{1}^{2} \alpha_{2}^{2}}} \cos \xi \\
\beta_{2}=\frac{\partial S}{\partial \alpha_{2}}=-\int_{0}^{\psi} \frac{\alpha_{2} d \psi}{\sqrt{\alpha_{4}^{2}-\alpha_{2}^{2} \operatorname{ch}^{-2} \psi}}+\int_{\rho_{\min }}^{\rho} \frac{d\left(\alpha_{2} / \rho-G / \alpha_{2}\right)}{\sqrt{-G^{2} / \alpha_{1}^{2}+2 G / \rho-\alpha_{2}^{2} / \rho^{2}}}
\end{gathered}
$$

и аналогично получим

$$
\beta_{2}=\frac{\partial S}{\partial \alpha_{2}}=-\arcsin \left(\frac{\alpha_{2}}{\sqrt{\alpha_{4}^{2}-\alpha_{2}^{2}}} \operatorname{sh} \psi\right)+\xi, \quad \operatorname{sh} \lambda \sin \left(\beta_{2}-\xi\right)=\operatorname{sh} \psi .
$$

То же самое получится, если спроектировать (17) на $\mathbf{e}_{4}$ :

$\operatorname{sh} \psi=\operatorname{sh} \lambda \sin \nu$.

Стало быть, $\nu=\beta_{2}+\xi$, так что уравнение (18) дает уравнение эллипса:

$$
\rho=\frac{p}{1+e \cos \left(\nu-\beta_{2}\right)}, \quad p=\frac{\alpha_{2}^{2}}{G}, \quad e=\sqrt{1-\left(\frac{\alpha_{2}}{\alpha_{1}}\right)^{2}} .
$$


Последний угол будем рассматривать так же, как в классической задаче. Запишем ключевые формулы:

$$
\begin{gathered}
H=-\frac{G^{2}}{2 \alpha_{1}^{2}}, \quad d \beta_{1}=\frac{\alpha_{2}^{3}}{\alpha_{1}^{3}} \frac{d \xi}{(1+e \cos \xi)^{2}}, \quad \beta_{1}=E-e \sin E \\
\cos \xi=\frac{\cos E-e}{1-e \cos E}, \quad \sin \xi=\frac{\sqrt{1-e^{2}} \sin E}{1-e \cos E}, \quad d \xi=\frac{\sqrt{1-e^{2}}}{1-e \cos E} .
\end{gathered}
$$

После проектирования эллипса (19) из $\mathbb{R}^{2,1}$ в плоскость видимых движений $\mathbb{R}^{2}\{x, y\}$ его фокус, вообще говоря, уже не будет попадать в притягивающий центр. Но это смещение несущественно, если величина $\lambda$ мала, т. е. $\lambda^{2}=0$.

\section{5. ВЫБОР ПОСТОЯННОЙ ЭНЕРГИИ И СМЫСЛ "МЕТАВРЕМЕНИ"}

Теория, рассмотренная в работах [7], [8], приводит к пониманию физического смысла величины $s$. В самом деле, поскольку $\sum\left(\partial L / \partial t_{\varkappa}\right) \equiv 0$, первый интеграл "импульса по времени" $\sum\left(\partial L / \partial t_{\nu}^{\prime}\right)=$ const, или $(1 / M) \sum m_{\nu} t_{\nu}^{\prime}=k$, причем от безразмерной величины $k$ движение относительно центра масс не зависит. Отсюда

$$
s=\frac{1}{k} \frac{\sum m_{\nu} t_{\nu}}{M}
$$

Если применить преобразование Лоренца (9), то величина $s$ должна быть заменена на

$$
\tilde{s}=s+\frac{X}{k},
$$

т. е. происходит изменение начала отсчета.

Нельзя вычислять $s$ в инерциальной системе, связанной с центром масс: получится, что $k=0$. Стало быть, определение $s$ применительно к Солнечной системе связано как минимум с ее движением относительно Галактики.

В работе [8] было доказано, что если постоянной энергии придать одно вполне определенное значение, а именно

$$
h=-\frac{M c^{2}}{2},
$$

то, во-первых, в динамике одной частицы в электромагнитном поле вариационный принцип Якоби приводит к общепринятым формулам (см., например, книгу [13]), а независимая переменная приравнивается к собственному времени, и, во-вторых, в динамике нескольких взаимодействующих частиц облегчается выбор независимой переменной рассматриваемой теории (и уточняется переход к ньютоновой механике).

Приведем подробности. Для “чисто классических" решений $t_{\nu}=t_{\varkappa} \equiv \tau$, тогда $\tau=k s$, так что в кинетическую энергию входит слагаемое

$$
-\frac{1}{2} c^{2} \sum m_{\nu} t_{\nu}^{\prime 2}=-\frac{k^{2} M c^{2}}{2} .
$$

Если принять условие (22), то получим

$$
k^{2}\left(\frac{1}{2} \sum m_{\nu}\left(\frac{d r_{\nu}}{d \tau}\right)^{2}\right)+V=\left(k^{2}-1\right) \frac{M c^{2}}{2} .
$$


Заменив $V / k^{2}$ на $V^{*}$, получим классический интеграл энергии в виде

$$
T+V^{*}=\frac{k^{2}-1}{k^{2}} \frac{M c^{2}}{2}=h^{*}= \pm \frac{M u^{2}}{2},
$$

где $u$ - характерный параметр с размерностью скорости. Если в качестве инерциальной взять систему координат с началом в центре масс Солнечной системы и осями, ориентированными по звездам, то получим $h^{*}<0$ и $u / c$ порядка $10^{-4}$. Если считать Галактику вращающейся, то $h^{*}>0$ и $u / c$ имеет порядок $10^{-3}$ (численные данные можно найти в учебнике [21]). Так или иначе, $k$ примерно равно единице. Для движений, близких к классическим, справедлив тот же вывод.

\section{6. КИНЕМАТИКА (ПСЕВДО)ТВЕРДОГО ТЕЛА}

Пусть $D^{c}=\operatorname{diag}\left(1,1,1,-c^{2}\right)$. Это матрица Грама координатного базиса. Очевидно, что матрица $Q(s)$ задает изометрию псевдоевклидовой метрики, т. е.

$$
\langle\mathbf{a}, \mathbf{b}\rangle=\langle Q(s) \mathbf{a}, Q(s) \mathbf{b}\rangle=\left(Q(s) \mathbf{a}, D^{c} Q(s) \mathbf{b}\right),
$$

где $(\cdot, \cdot)$ - формальное скалярное произведение, тогда и только тогда, когда

$$
Q^{*} D^{c} Q=D^{c} \Longleftrightarrow Q^{*} D^{c}=D^{c} Q^{-1} \Longleftrightarrow Q^{*}=D^{c} Q^{-1} D^{c^{-1}} .
$$

Дифференцируя формулу (23), получим

$$
0=\left\langle Q^{\prime}(s) \mathbf{a}, Q(s) \mathbf{b}\right\rangle+\left\langle Q(s) \mathbf{a}, Q^{\prime}(s) \mathbf{b}\right\rangle=\left(Q(s) \mathbf{a}, D^{c} Q^{\prime}(s) \mathbf{b}\right)+\left(Q^{\prime}(s) \mathbf{a}, D^{c} Q(s) \mathbf{b}\right) .
$$

Отсюда и из соотношения (24) вытекает, что производная матрицы $Q(s)$ удовлетворяет любому из равносильных условий

$$
\frac{d Q}{d s}=D^{c^{-1}} W^{c} Q, \quad \frac{d Q}{d s}=Q D^{c^{-1}} W^{c},
$$

где $W^{c}$ - кососимметрическая матрица.

Кососимметрический тензор угловой скорости. Соответствие $W^{c} \mapsto \widehat{W}^{c}=$ $D^{c^{-1}} W^{c}$ из формул (25) запишем подробнее:

$$
W^{c}=\left(\begin{array}{cccc}
0 & -\omega_{3} & \omega_{2} & c \omega_{4} \\
\omega_{3} & 0 & -\omega_{1} & c \omega_{5} \\
-\omega_{2} & \omega_{1} & 0 & c \omega_{6} \\
-c \omega_{4} & -c \omega_{5} & -c \omega_{6} & 0
\end{array}\right) \mapsto \widehat{W}^{c}=\left(\begin{array}{cccc}
0 & -\omega_{3} & \omega_{2} & c \omega_{4} \\
\omega_{3} & 0 & -\omega_{1} & c \omega_{5} \\
-\omega_{2} & \omega_{1} & 0 & c \omega_{6} \\
\omega_{4} / c & \omega_{5} / c & \omega_{6} / c & 0
\end{array}\right) .
$$

Пусть $\boldsymbol{\sigma}(s)$ - любой вектор, жестко связанный с телом. Тогда

$$
\frac{d}{d s} \boldsymbol{\sigma}(s)=\widehat{W}^{c} \boldsymbol{\sigma}(s) .
$$

По смыслу величины $\omega_{k}=\omega_{k}(s)$ аналогичны классическим компонентам угловой скорости в неподвижном репере, но их шесть, а не четыре (это компоненты бивектора). Произвольное равномерное вращение $\left(\omega_{k}=\mathrm{const}\right)$ порождается векторным полем

$$
\mathbf{v}(\mathbf{q})=\sum \omega_{k} \overleftarrow{F}_{k}(\mathbf{q})
$$

5 Теоретическая и математическая физика, т. 166, № 3, 2011 г. 
Перейдем к тензорным переменным, т. е. и к $c=1$. Напишем $W^{1}=\sum \omega_{k} W_{k}^{1}$, где $W_{k}^{1}$ - очевидные базисные кососимметрические матрицы из нулей и единиц.

Теперь рассмотрим первую из формул (25). Введем в употребление подвижный репер. Отметим какое-нибудь одно "начальное" положение твердого тела в имеющейся системе отсчета и для каждого жестко связанного с телом вектора напишем $\boldsymbol{\sigma}(s)=Q(s) \mathbf{c}$, т. е. представим его как результат некоторого (псевдо)поворота. Тогда

$$
\begin{gathered}
\mathbf{c}=(\mathbf{d}, \delta), \quad \mathbf{d}^{2}-\delta^{2}=l^{2}=\mathrm{const} \\
\boldsymbol{\sigma}^{\prime}(s)=Q^{\prime}(s) \mathbf{c}=Q(s) Q^{-1}(s) Q^{\prime}(s) \mathbf{c}=Q(s) \widehat{W}^{1}(s) \mathbf{c} \\
\left\langle\boldsymbol{\sigma}^{\prime}, \boldsymbol{\sigma}^{\prime}\right\rangle=\left\langle Q \widehat{W}^{1} \mathbf{c}, Q \widehat{W}^{1} \mathbf{c}\right\rangle=\left\langle\widehat{W}^{1} \mathbf{c}, \widehat{W}^{1} \mathbf{c}\right\rangle .
\end{gathered}
$$

Вектор $\widehat{W}^{1} \mathbf{c}$ составлен из компонент вектора скорости в подвижном репере $\mathbf{q}_{\beta}(s)=$ $Q(s) \mathbf{e}_{\beta}$.

Скобки Пуассона и векторные поля. На гладком многообразии с локальными координатами $q_{1}, \ldots, q_{n}$ векторное поле записывается в виде $\boldsymbol{a}=\sum_{i=1}^{k} a_{i} \partial / \partial q_{i}$ и тем самым задает дифференцирование гладких функций $f(q)$ вдоль этого вектора или поля, а именно

$$
\boldsymbol{a}(f)=\sum_{i} a_{i}(q) \frac{\partial f}{\partial q_{i}} .
$$

Синонимами являются: дифференциальный оператор, инфинитезимальный оператор, генератор однопараметрической группы (фазового потока). Обозначим

$$
P_{a}=\sum p_{i} a_{i}(q), \quad P_{b}=\sum p_{j} b_{j}(q),
$$

тогда, если $\boldsymbol{a}$ и $\boldsymbol{b}$ - два поля, то их скобка Ли (коммутатор) $[\boldsymbol{a}, \boldsymbol{b}](f)=\boldsymbol{a}(\boldsymbol{b}(f))-$ $\boldsymbol{b}(\boldsymbol{a}(f))$ есть новое векторное поле. Для нас важно, что

$$
\boldsymbol{a}(f) \equiv\left\{f, P_{\boldsymbol{a}}\right\}, \quad\left\{P_{\boldsymbol{a}}, P_{\boldsymbol{b}}\right\} \equiv-P_{[\boldsymbol{a}, \boldsymbol{b}]} .
$$

Здесь минус появляется вследствие строгого соблюдения традиций в определениях $(\{p, q\}=-1)$.

Разница между правыми и левыми сдвигами. Пусть $A, B$ - постоянные матрицы. Пусть есть также два дифференциальных уравнения вида $d M / d t_{1}=M A$, $d M / d t_{2}=M B$ и, стало быть, два векторных поля $\boldsymbol{a}, \boldsymbol{b}$ на пространстве квадратных матриц $\{M\}$. Тогда коммутатор $[\boldsymbol{a}, \boldsymbol{b}]$ точно так же порождается коммутатором матриц $[A, B]=A B-B A$. Если же мы имеем два уравнения вида $d M / d t_{1}=A M$, $d M / d t_{2}=B M$, то коммутатор соответствующих полей $[\boldsymbol{a}, \boldsymbol{b}]$ точно так же порождается коммутатором матриц $[A, B]$, взятым со знаком минус.

Группа $S O(3,1)$. Отождествим положения тела и матрицы $Q$, образующие группу $S O(3,1)$. Первая из формул $(25)$ задает на $S O(3,1)$ векторные поля $\boldsymbol{L}_{k}$, в силу которых $Q^{\prime}(s)=\widehat{W}_{k}^{1} Q(s)$. Это поля, порождающие левые сдвиги на $S O(3,1)$. Вторая из формул $(25)$ задает на $S O(3,1)$ векторные поля $\boldsymbol{R}_{k}$, в силу которых $Q^{\prime}(s)=Q(s) \widehat{W}_{k}^{1}$. Это поля, порождающие правые сдвиги на $S O(3,1)$. Можно написать соответственно $Q^{\prime}(s)=\sum^{k} \omega_{k} \boldsymbol{L}_{k}(Q)$ или $Q^{\prime}(s)=\sum \omega_{k} \boldsymbol{R}_{k}(Q)$. 
Коммутаторы полей $\boldsymbol{L}_{k}$ такие же, как у полей $\overleftarrow{F}_{k}$, которые действуют на каждую точку тела и тем самым задают действие на $S O(3,1)$.

Коммутаторы полей $\boldsymbol{R}_{k}$ отличаются от коммутаторов $\boldsymbol{L}_{k}$ знаком; стало быть, в силу формулы (29) (там есть минус) формируются по тому же правилу (10), что и скобки Пуассона функций $F_{k}$. Согласно формулам $(28)$ поставим полям $\boldsymbol{R}_{k}$ в соответствие линейные гамильтонианы $P_{k}$. Тогда их скобки знаком отличаются от (10): попарные скобки Пуассона

$$
\begin{array}{ll}
\left\{P_{i+1}, P_{i+2}\right\}=-P_{i}, & \left\{P_{i+1}, P_{i+5}\right\}=-P_{i+4}, \\
\left\{P_{i+1}, P_{i+6}\right\}=P_{i+4}, & \left\{P_{i+5}, P_{i+6}\right\}=P_{i}
\end{array}
$$

при $i=1,2,3(\bmod 3)$. Далее мы объясним, почему эти формулы имеют такое значение.

\section{7. НОВАЯ ФОРМА УРАВНЕНИЙ ДВИЖЕНИЯ СО СКОБКАМИ ПУАССОНА}

Кратко напомним общие положения [15], [16]: неголономная система (или голономная в псевдоскоростях, как у нас) без непотенциальных сил задана, если выписаны лагранжиан и связи:

$$
L(\dot{q}, q, t), \quad \dot{q}_{i}=v_{i}\left(u_{1}, \ldots, u_{m}, q_{1}, \ldots, q_{n}, t\right)
$$

и действует следующее соглашение об индексах:

$$
i, j, k=1, \ldots, n, \quad \lambda, \mu, \nu=1, \ldots, m \leqslant n, \quad s, r=m+1, \ldots, n .
$$

Здесь $m$ есть число степеней свободы. Пусть $(\cdot) *$ означает подстановку выражений для скоростей $\dot{q}$ через псевдоскорости $u$.

Вводятся тройственная характеристическая функиия

$$
Y(t, u ; q ; p)=\left[L-\sum p_{i} \dot{q}_{i}\right]^{*}
$$

и псевдополная производная по времени

$$
\frac{d^{u}}{d t} \equiv \frac{\partial}{\partial t}+\sum \dot{u}_{\lambda} \frac{\partial}{\partial u_{\lambda}} .
$$

Тогда для получения динамических уравнений надо записать

$$
\frac{d^{u}}{d t} \frac{\partial Y}{\partial u_{\lambda}}=\left\{\frac{\partial Y}{\partial u_{\lambda}}, Y\right\}
$$

и только после этого подставить вместо $p_{j}$ выражения

$$
p_{j}=\left(\frac{\partial L}{\partial \dot{q}_{j}}\right)^{*} \text {. }
$$

Это обобщенные импульсы, выраженные через псевдоскорости. 
Обычная неголономная система задается следующим образом:

$$
\begin{aligned}
L=T-V & =\frac{1}{2} \sum a_{j k} \dot{q}_{i} \dot{q}_{k}-V, \\
\frac{d q_{i}}{d t} & =\sum \epsilon_{i \lambda}(q) u_{\lambda} ;
\end{aligned}
$$

кинетическая энергия с учетом связей имеет вид

$$
T^{*}(u, q)=\frac{1}{2} \sum g_{\lambda \mu}(q) u_{\lambda} u_{\mu} .
$$

УТВЕРЖДЕНИЕ [16]. После того как переменные q, и выбраны, все известные способы составления уравнений движения приводят к одному результату:

$$
\sum_{\mu} g_{\lambda \mu}(q) \dot{u}_{\mu}+\sum_{\mu \nu} G_{\lambda, \mu \nu}(q) u_{\mu} u_{\nu}+\sum_{i} \epsilon_{i \lambda}(q) \frac{\partial V}{\partial q_{i}}=0 .
$$

Разница заключается лишь в программах вычисления коэффициентов $G_{\lambda, \mu \nu}=$ $G_{\lambda, \nu \mu}$. Новый общий способ для нашего случая принимает следующий вид: выписываем $P_{\nu}=\sum p_{j} \epsilon_{j \nu}(q)$ - функции вида (29). Тогда уравнение (34) получается в два этапа: сначала выписываем

$$
\frac{d}{d t} \frac{\partial L^{*}}{\partial u_{\lambda}}+\left\{P_{\lambda}, L^{*}\right\}=\left\{P_{\lambda}, \sum u_{\mu} P_{\mu}\right\}
$$

(здесь и берутся скобки Пуассона в переменных $p, q$ ) и только после этого подставляем выражения (31). Правая часть формулы (35) порождает члены неголономности.

В случае голономной системы эти члены являются мерой отличия подвижного репера от координатного.

Итак, для действительно неголономной системы уравнения движения, вообще говоря, не могут определяться одной только функцией $L^{*}$. Для голономных систем, наоборот, если разные лагранжианы $L$ на связи порождают один и тот же $L^{*}$, то и уравнения движения будут одинаковыми. Ниже мы увидим, как новая форма уравнений движения позволяет воспользоваться этим соображением.

\section{8. КИНЕТИЧЕСКАЯ ЭНЕРГИЯ ВРАЩЕНИЯ}

Соотношение $Q^{\prime}(s)=Q(s) \widehat{W}^{1}(s)$ приводит к выражениям вида $(33)$ :

$$
\dot{\mathbf{q}}_{i}=-\omega_{i+1} \mathbf{q}_{i+2}+\omega_{i+2} \mathbf{q}_{i+1}+\Omega_{i} \mathbf{q}_{4}, \quad \dot{\mathbf{q}}_{4}=\Omega_{1} \mathbf{q}_{1}+\Omega_{2} \mathbf{q}_{2}+\Omega_{3} \mathbf{q}_{3},
$$

в которых индексы, напомним, принимают значения $1,2,3(\bmod 3)$. Положим

$$
\begin{gathered}
\mathbf{c}=(\mathbf{d}, \delta), \quad \mathbf{d}^{2}-\delta^{2}=l^{2}=\mathrm{const}, \quad \Omega_{i}=\omega_{3+i}, \\
\boldsymbol{\omega}=\left(\omega_{1}, \omega_{2}, \omega_{3}\right), \quad \boldsymbol{\Omega}=\left(\Omega_{1}, \Omega_{2}, \Omega_{3}\right)
\end{gathered}
$$

и получим в подвижном репере

$$
\begin{aligned}
\boldsymbol{\sigma}_{\nu}^{\prime} & =\left(\left[\boldsymbol{\omega}, \mathbf{d}_{\nu}\right]+\delta_{\nu} \boldsymbol{\Omega},\left(\boldsymbol{\Omega}, \mathbf{d}_{\nu}\right)\right), \\
2 \mathcal{T}_{\text {вращ }} & =\sum_{\nu}^{2} m_{\nu}\left\{\left(\left[\boldsymbol{\omega}, \mathbf{d}_{\nu}\right]+\delta_{\nu} \boldsymbol{\Omega}\right)^{2}-\left(\boldsymbol{\Omega}, \mathbf{d}_{\nu}\right)^{2}\right\}= \\
& =\sum^{2} m_{\nu}\left[\boldsymbol{\omega}, \mathbf{d}_{\nu}\right]^{2}+\sum^{2} m_{\nu} \delta_{\nu}\left([\boldsymbol{\Omega}, \boldsymbol{\omega}], \mathbf{d}_{\nu}\right)+\sum^{2} m_{\nu} \delta_{\nu}^{2} \boldsymbol{\Omega}^{2}-\sum^{2} m_{\nu}\left(\boldsymbol{\Omega}, \mathbf{d}_{\nu}\right)^{2} .
\end{aligned}
$$


В начальном положении введем произведения инерции

$$
M_{2} I^{\alpha \beta}=\sum^{2} m_{\nu} \xi_{\nu}^{\alpha} \xi_{\nu}^{\beta}, \quad \alpha, \beta=1,2,3,4 .
$$

Сейчас у нас нет возможности отвлекаться на крайне интересную динамику “твердого тела" по инерции (расширение классического случая Эйлера на задачу о вращении твердого тела), так что упростим себе задачу и предположим тело максимально изотропным относительно пространственных поворотов (это и есть шар), так что $I^{\alpha \beta}=0$ при $\alpha \neq \beta$ и $I^{11}=I^{22}=I^{33}=I, I^{44}=I^{\prime}$. Это определение, разумеется, не выдерживает преобразований Лоренца, т. е. может быть верным только в специально подобранной системе координат.

В сделанных предположениях

$$
2 \mathcal{T}_{\text {вращ }}^{*}=2 I \omega^{2}+\left(I^{\prime}-I\right) \boldsymbol{\Omega}^{2} .
$$

\section{9. ГРАВИТАЦИОННЫЙ ПОТЕНЦИАЛ}

Потециальная энергия гравитационного взаимодействия для системы "тело-точка" имеет вид

$$
V=-f \sum^{2} \frac{m_{1} m_{\nu}}{\Delta_{1 \nu}}=-\mu \sum^{2} \frac{f\left(m_{1}+M_{2}\right) m_{\nu}}{M_{2} \sqrt{\left\langle\mathbf{q}_{\nu}-\mathbf{q}_{1}, \mathbf{q}_{\nu}-\mathbf{q}_{1}\right\rangle}}
$$

Поскольку $\mathbf{q}_{\nu}-\mathbf{q}_{1}=\boldsymbol{\sigma}_{\nu}-\mathbf{q}$, то

$$
\left\langle\mathbf{q}_{\nu}-\mathbf{q}_{1}, \mathbf{q}_{\nu}-\mathbf{q}_{1}\right\rangle=\left\langle\boldsymbol{\sigma}_{\nu}-\mathbf{q}, \boldsymbol{\sigma}_{\nu}-\mathbf{q}\right\rangle=\langle\mathbf{q}, \mathbf{q}\rangle-2\left\langle\mathbf{q}, \boldsymbol{\sigma}_{\nu}\right\rangle+\left\langle\boldsymbol{\sigma}_{\nu}, \boldsymbol{\sigma}_{\nu}\right\rangle .
$$

Положим $\mathbf{q}=\rho \mathbf{e},\langle\mathbf{e}, \mathbf{e}\rangle=1$ и $G=f\left(m_{1}+M_{2}\right)$. Тогда

$$
\begin{aligned}
V & =-\frac{\mu G}{\rho} \sum^{2} \frac{m_{\nu}}{M_{2} \sqrt{1-2\left\langle\mathbf{e}, \boldsymbol{\sigma}_{\nu}\right\rangle / \rho+\left\langle\boldsymbol{\sigma}_{\nu}, \boldsymbol{\sigma}_{\nu}\right\rangle / \rho^{2}}}= \\
& =-\frac{\mu G}{\rho}\left(1+\frac{1}{2 M_{2} \rho^{2}} \sum^{2} m_{\nu}\left[-\left\langle\boldsymbol{\sigma}_{\nu}, \boldsymbol{\sigma}_{\nu}\right\rangle+3\left\langle\mathbf{e}, \boldsymbol{\sigma}_{\nu}\right\rangle^{2}\right]+O\left(\frac{1}{\rho^{3}}\right)\right) .
\end{aligned}
$$

Для разложения потенциала применим подвижный репер, так как только в этом случае есть шанс воспользоваться постоянными моментами инерции. Итак, $\mathbf{q}=\rho \mathbf{e}$, $\mathbf{e}=\sum \gamma_{\beta} \mathbf{e}_{\beta}, \gamma_{1}^{2}+\gamma_{2}^{2}+\gamma_{3}^{2}-\gamma_{4}^{2}=1$.

Опустим вычисления (очень похожие на соответствующие случаю с кинетической энергией) и с ошибкой $O\left(1 / \rho^{4}\right)$ получим

$$
V=-\frac{\mu G}{\rho}\left(1+\frac{1}{2 \rho^{2}}\left[I^{\prime}+\left(I+I^{\prime}\right) \gamma_{4}^{2}\right]\right) \text {. }
$$

Величина $\gamma_{4}^{2}$ зависит и от ориентации тела $Q$, и от направления q. Поэтому легко может оказаться, что вращение тела и орбитальное движение влияют друг на друга. Мы будем искать решения, когда подобного не происходит. 


\section{0. ПРИМЕНЕНИЕ НОВОЙ ФОРМЫ УРАВНЕНИЙ ДВИЖЕНИЯ}

Лагранжиан. Без слагаемого для общего центра масс лагранжиан имеет вид

$$
L^{*}=\frac{\mu\left\langle\mathbf{q}^{\prime}, \mathbf{q}^{\prime}\right\rangle}{2}+I \boldsymbol{\omega}^{2}+\frac{I^{\prime}-I}{2 \Omega^{2}}+\frac{\mu G}{\rho}\left(1+\frac{1}{2 \rho^{2}}\left[I^{\prime}+\left(I+I^{\prime}\right) \boldsymbol{\gamma}_{4}^{2}\right]\right) .
$$

В системе “тело-точка" мы используем, во-первых, простейшие координаты и импульсы

$$
\left(x^{1}, x^{2}, x^{3}, x^{4}\right)=\mathbf{q}, \quad\left(p^{1}, p^{2}, p^{3}, p^{4}\right)=\mathbf{p} .
$$

Во-вторых, положение на $S O(3,1)$ станем задавать избыточными координатами элементами $q_{\alpha \beta}$ матрицы $Q$ (тогда соотношение (24) задает связи между координатами, и, кроме того, надо потребовать, чтобы $\operatorname{det} Q>0)$. Столбцы матрицы $Q$ образованы компонентами векторов подвижного репера тела в исходной системе координат, эти столбцы у нас обозначены $\mathbf{q}_{\beta}$.

Выражения (36) уже имеют вид (33) с псевдоскоростями $\omega_{1}, \ldots, \omega_{6}$. Из интегральных многообразий этого распределения плоскостей над 16-мерным пространством координат $q_{\alpha \beta}$ необходимо взять то, которое проходит через единичную матрицу. Получится $S O(3,1)$.

Координатам $q_{\alpha \beta}$ соответствуют канонические импульсы $p_{\alpha \beta}$, которые мы сведем в векторы $\mathbf{p}_{\beta}$. Компонентами $\boldsymbol{R}_{k}^{\alpha \beta}$ векторных полей $\boldsymbol{R}_{k}$ служат элементы матрицы $W_{k}^{1}$. Полям $\boldsymbol{R}_{k}$ соответствуют линейные по импульсам функции $P_{k}=\sum p_{\alpha \beta} \boldsymbol{R}_{k}^{\alpha \beta}$. Их коммутаторы выписаны выше.

Поскольку в избыточных переменных $\sum p_{\alpha \beta} \dot{q}_{\alpha \beta}=\sum^{k}\left(\mathbf{p}_{\beta}, \dot{\mathbf{q}}_{\beta}\right)$, из выражений (36) получаем

$$
\begin{gathered}
\sum\left(\mathbf{p}_{\beta}, \dot{\mathbf{q}}_{\beta}\right)=\sum \omega_{i}\left(\left(\mathbf{p}_{i+2}, \mathbf{q}_{i+1}\right)-\left(\mathbf{p}_{i+1}, \mathbf{q}_{i+2}\right)\right)+\sum \Omega_{i}\left(\left(\mathbf{p}_{i}, \mathbf{q}_{4}\right)+\left(\mathbf{p}_{4}, \mathbf{q}_{i}\right)\right), \\
P_{i}=\left(\mathbf{p}_{i+2}, \mathbf{q}_{i+1}\right)-\left(\mathbf{p}_{i+1}, \mathbf{q}_{i+2}\right), \quad P_{3+i}=\left(\mathbf{p}_{i}, \mathbf{q}_{4}\right)-\left(\mathbf{p}_{4}, \mathbf{q}_{i}\right),
\end{gathered}
$$

а также

$$
\omega_{i}=\left\langle\mathbf{q}_{i+1}^{\prime}, \mathbf{q}_{i+2}\right\rangle=-\left\langle\mathbf{q}_{i+2}^{\prime}, \mathbf{q}_{i+1}\right\rangle, \quad \Omega_{i}=\omega_{3+i}=\left\langle\mathbf{q}_{4}^{\prime}, \mathbf{q}_{i}\right\rangle=-\left\langle\mathbf{q}_{i}^{\prime}, \mathbf{q}_{4}\right\rangle .
$$

Чтобы в удобном для применения теории виде задать кинетическую энергию, используем формулы (39) и вспомним, что до подстановки псевдоскоростей мы имели

$$
L=\frac{\mu\left\langle\mathbf{q}^{\prime}, \mathbf{q}^{\prime}\right\rangle}{2}+T_{\text {вращ }}, \quad 2 \mathcal{T}_{\text {вращ }}=2 I \sum\left\langle\dot{\mathbf{q}}_{i+1}, \mathbf{q}_{i+2}\right\rangle^{2}+\left(I^{\prime}-I\right) \sum\left\langle\mathbf{q}_{4}^{\prime}, \mathbf{q}_{i}\right\rangle^{2} .
$$

Эта квадратичная форма 16 скоростей вырожденна, но для динамики тела важна

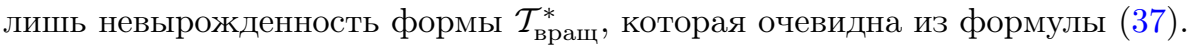

Ясно, что импульсы $\mathbf{p}=\left(p^{1}, p^{2}, p^{3}, p^{4}\right)$ коммутируют между собой и с $P_{k}$. Набор псевдоскоростей здесь имеет вид $u=(\dot{\mathbf{q}}, \boldsymbol{\omega}, \boldsymbol{\Omega})$, так что из лагранжиана (40) следует, что

$$
\begin{gathered}
\mathbf{p}^{*}=\mu \dot{\mathbf{q}}, \quad \mathbf{p}_{i+1}^{*}=2 I \omega_{i} D^{1} \mathbf{q}_{i+2}, \quad \mathbf{p}_{4}^{*}=\left(I^{\prime}-I\right) \sum \Omega_{i} D^{1} \mathbf{q}_{i}, \\
P_{i}^{*}=\left(\mathbf{p}_{i+2}^{*}, \mathbf{q}_{i+1}\right)-\left(\mathbf{p}_{i+1}^{*}, \mathbf{q}_{i+2}\right)=\left\langle 2 I \omega_{i+1} \mathbf{q}_{i}, \mathbf{q}_{i+1}\right\rangle-\left\langle 2 I \omega_{i} \mathbf{q}_{i+2}, \mathbf{q}_{i+2}\right\rangle=-2 I \omega_{i}, \\
P_{i+3}^{*}=\left(\mathbf{p}_{i}^{*}, \mathbf{q}_{4}\right)+\left(\mathbf{p}_{4}^{*}, \mathbf{q}_{i}\right)=\left\langle 2 I \omega_{i+2} \mathbf{q}_{i+1}, \mathbf{q}_{4}\right\rangle+\left\langle\left(I^{\prime}-I\right) \sum \Omega_{j} \mathbf{q}_{j}, \mathbf{q}_{i}\right\rangle=\left(I^{\prime}-I\right) \Omega_{i} .
\end{gathered}
$$


Уравнения (35) после применения формул (30) приобретают вид

$$
\begin{gathered}
\mu \ddot{x}^{\alpha}-\frac{\partial V\left(\rho, \gamma_{4}^{2}\right)}{\partial \rho}\left\{p^{\alpha}, \rho\right\}=0, \\
2 I \dot{\omega}_{i}+(\ldots) \gamma_{4}\left\{P_{i}, \gamma_{4}\right\}=-\omega_{i+1} P_{i+2}+\omega_{i+2} P_{i+1}-\Omega_{i+1} P_{i+5}+\Omega_{i+2} P_{i+4}, \\
\left(I^{\prime}-I\right) \dot{\Omega}_{i}+(\ldots) \gamma_{4}\left\{P_{i+3}, \gamma_{4}\right\}=-\omega_{i+1} P_{i+5}+\omega_{i+2} P_{i+4}-\Omega_{i+1} P_{i+2}+\Omega_{i+2} P_{i+1} .
\end{gathered}
$$

Здесь многоточием обозначены множители, получающиеся при дифференцировании потенциала $V$; их детальный вид несуществен для дальнейшего. После подстановки формул $(31)$, т. е. учета выражений $P_{i}^{*}, P_{i+3}^{*}$, справа получатся нули в силу того, что мы рассматриваем шар.

Класс нужных частных движений. Теперь найдем движения, при которых величина $\gamma_{4}$ останется нулем - тогда движение точки $\mu$ отделится от вращения тела. Более того, потребуем, чтобы выполнялись условия $\mathbf{q}_{4}(s) \equiv \mathbf{e}_{4}$ и $\left\langle\mathbf{q}(s), \mathbf{e}_{4}\right\rangle \equiv 0$. Присоединяя последнее из уравнений (36), видим, что условие $\mathbf{q}_{4}(s) \equiv \mathbf{e}_{4}$ действительно выполняется при подходяших начальных условиях, если брать решения с $\Omega_{i} \equiv 0$. При этом можно считать $\omega_{i} \equiv$ const (равномерное вращение шара). Условие $\left\langle\mathbf{q}(s), \mathbf{e}_{4}\right\rangle \equiv 0$ означает, что следует использовать $\mathbf{q}(s)$ из классической подзадачи.

Попутно мы убедились в том, что уравнения движения тела допускают такие решения, при которых происходит только чистое вращение (собственные времена, которые при этом не изменяются, будут сохранять начальный разброс).

\section{1. ОСРЕДНЕНИЕ И ЭВОЛЮЦИЯ}

Зная, для каких начальных условий величина $\gamma_{4}$ остается равной нулю, мы можем теперь отбросить в потенциале слагаемое с $\gamma_{4}$ и для удобства рассмотреть получающуюся задачу с любыми начальными условиями. Тело в этом случае равномерно вращается, а изменение $\mathbf{q}(s)$ описывается гамильтонианом

$$
H=-\frac{G^{2}}{2 \alpha_{1}^{2}}+H_{1}, \quad H_{1}=\frac{G I^{\prime}}{\rho^{3}} .
$$

Нет необходимости выписывать выражение $H_{1}$ в переменных действие-угол. Зная, что невозмущенная система одночастотная (быстрая переменная только $\beta_{1}$ ), мы приме́ним метод осреднения [10] и заменим возмущение $H_{1}$ на его среднее:

$$
\begin{aligned}
\bar{H}_{1} & =\frac{1}{2 \pi} \int_{0}^{2 \pi} H_{1} d \beta_{1}=-\frac{G I^{\prime}}{2 \pi} \int_{0}^{2 \pi} \frac{1}{\rho^{3}} \frac{\alpha_{2}^{3}}{\alpha_{1}^{3}} \frac{d \xi}{(1+e \cos \xi)^{2}}= \\
& =-\frac{G I^{\prime}}{2 \pi} \frac{\alpha_{2}^{3}}{\alpha_{1}^{3}} \frac{G^{3}}{\alpha_{2}^{6}} \int_{0}^{2 \pi}(1+e \cos \xi) d \xi=-\frac{G^{4} I^{\prime}}{\alpha_{1}^{3} \alpha_{2}^{3}} .
\end{aligned}
$$

Следовательно, переменные $\alpha_{1}, \alpha_{2}, \alpha_{3}, \beta_{3}$ не эволюционируют, траектория представляет собой эллипс с полуосью $a=p\left(1-e^{2}\right)$, вся прецессия которого обеспечивается изменением угла $\beta_{2}$; за период обращения

$$
T=\frac{2 \pi \alpha_{1}^{3}}{G^{2}}=\frac{2 \pi \alpha^{3 / 2}}{\sqrt{G}}
$$


имеем

$$
\varepsilon=\delta \beta_{2}=\beta_{2}^{\prime} T=\frac{3 I^{\prime} G^{4}}{\alpha_{1}^{3} \alpha_{2}^{4}} \frac{2 \pi \alpha_{1}^{3}}{G^{2}}=6 \pi \frac{I^{\prime}}{\alpha_{2}^{2}} \frac{G^{2}}{\alpha_{2}^{4}}=6 \pi \frac{I^{\prime}}{p^{2}},
$$

где используется фокальный параметр $p=a\left(1-e^{2}\right)$.

\section{2. КОММЕНТАРИИ}

Прецессия орбиты Меркурия составляет около 5600” в столетие, 5557" из них объясняются классической теорией тяготения вместе с теорией возмущений, а недостающие $43^{\prime \prime}$ составляют релятивистское смещение. Такова общепринятая картина (более точные цифры и научный контекст см. в работах [22], [23], развернутую историю вопроса - в монографии [24]). Ключевой здесь является формула Эйнштейна, про которую Синг пишет [12]: "Ее престиж настолько велик, что вряд ли какая-нибудь теория гравитации может оказаться приемлемой, если она не будет давать эту (или практически неотличимую от нее) формулу". Вот эта формула в трех вариантах:

$$
\varepsilon=\frac{24 \pi^{3} a^{2}}{c^{2} T^{2}\left(1-e^{2}\right)}=6 \pi \frac{1}{c^{2}} \frac{G^{2}}{\alpha_{2}^{2}}=6 \pi \frac{G}{c^{2} p} .
$$

Синг пишет далее: “... смесь ньютоновской и эйнштейновской теорий психологически неприятна, ибо эти теории основываются на слишком разных исходных концепциях. Ситуация прояснится лишь после того, как будет решена рациональным и математически удовлетворительным образом релятивистская проблема многих тел". Модель Козлова-Никишина вполне способна послужить основой для этого, так как в ней классическая модель - не предел, а подмножество решений; все методы небесной механики [9] в принципе можно расширить, добавив каждой точке еще одну степень свободы - по временно́й координате - и сохраняя прежний математический аппарат.

На этом пути связи между классикой и современными теориями тяготения, построенными на языке римановой геометрии, могут оказаться более обстоятельными и богатыми деталями. Здесь уместно вспомнить мысль Пуанкаре о том, что динамику одной и той же системы можно описывать на основе разных геометрий, хотя бы только с выражением надежды на будущие исследования.

Укажем попутно на работу [25], в которой обобщается и подробно обсуждается попытка Пуанкаре написать формулу для релятивистского гравитационного взаимодействия двух материальных точек (оно зависело и от скоростей точек). Расхожее мнение, что подход Пуанкаре позволяет получить лишь часть прецессии, опровергается. Пуанкаре предложил целый класс возможных лоренц-инвариантных выражений для сил, но сам воспользовался только одним из возможных выражений. В работе [25] показано, что другой выбор позволяет получить правильные выражения для прецессии, отклонения светового луча, задержки радиолокационных сигналов и красного смещения.

Трудности с объяснением поведения Меркурия не могут быть объяснены на основе СГМ, если рассматривать его и Солнце как материальные точки. Но если одну из точек заменить на шар, то сразу отбросить такую возможность уже нельзя, например формула (41) даст для Меркурия величину, равную (42), если окажется, что $I^{\prime}=\alpha_{2}^{2} / c^{2}=G p / c^{2}$. Характер влияния (через члены порядка $1 / \rho^{3}$ в потенциале) 
аналитически такой же, как у сжатия Солнца, если оно имеет место (подробно об этом вопросе см. монографию [22]); однако теперь причиной эволюции является дисперсия собственных времен точек шара-Солнца (при фиксированной независимой переменной $s)$.

Принципиальная возможность объяснения эволюции посредством СГМ - главный вывод настоящей работы, но это только первый шаг. В дальнейшем нужно будет отказаться от нашего специального выбора начальных условий, при котором, в частности, орбитальное движение является классическим (т. е. нет расхождения между собственными временами центра масс шара и точки, что выглядит непоследовательно на фоне разброса собственных времен точек шара). Строгости ради мы не стали брать за основу ограниченную постановку задачи (шар притягивает точку, но не наоборот), но вычисления в ней показывают, что расхождение времен, если оно не слишком велико, вносит отрицательный вклад в скорость эволюции. Компенсировать расхождение формул (41) и (42) при применении к нескольким планетам можно попробовать рассмотрением задачи многих тел. Так, вычисления для круговой ограниченной задачи трех тел показывают, что добавочная прецессия мала, как и мнимое наклонение орбиты.

Итак, для СГМ характерно появление в теории многих параметров, которыми можно распоряжаться для движения в сторону конечной цели небесной механики по Пуанкаре.

\section{Список литературы}

[1] А. Пуанкаре, Новъе методы небесной механики, В кн. Избр. труды, т. 1, 2, Наука, М., 1971-1972.

[2] Г. А. Лоренц, А. Пуанкаре, А. Эйнштейн, Г. Минковский, Принцип относительности, Сборник статей, ОНТИ, М., 1935.

[3] Дж. Синг, Классическая динамика, Физматгиз, М., 1963.

[4] П. Дирак, "Обобщенная гамильтонова динамика", Вариационнъе принципъ механики, ред. Л. С. Полак, Физматгиз, М., 1959, 703-722.

[5] П. А. Николов, И. Т. Тодоров, ЭЧАЯ, 14:5 (1983), 1092-1111.

[6] В. В. Козлов, Е. М. Никишин, Вестн. Моск. ун-та. Сер. матем., механ., 1986, №5, $11-20$.

[7] Е. М. Никишин, "Свободная гамильтонова механика в $M^{4}$ и специальная теория относительности”, Труды семинара по векторному и тензорному анализу, 24, МГУ, М., 1988, 141-164.

[8] Я. В. Татаринов, А. В. Черкаев, Вестн. Моск. ун-та. Сер. матем., механ., 2007, №3, $59-63$.

[9] К. Шарлье, Небесная механика, Наука, М., 1966.

[10] В. И. Арнольд, В. В. Козлов, А. И. Нейштадт, "Математические аспекты классической и небесной механики", Динамические системь - 3, Итоги науки и техн. Сер. Соврем. пробл. мат. Фундам. направления, 3, ред. Р. В. Гамкрелидзе, ВИНИТИ, М., 1985, 5-290.

[11] В. Паули, Теория относительности, Наука, М., 1991.

[12] Дж. Синг, Общая теория относительности, ИЛ, М., 1963.

[13] Я. П. Терлецкий, Ю. П. Рыбаков, Электродинамика, Высшая школа, М., 1980.

[14] Б. А. Дубровин, С. П. Новиков, А. Т. Фоменко, Современная геометрия. Методъ и приложения, т. 2: Геометрия и топология многообразий, Эдиториал УРСС, М., 1986.

[15] Я. В. Татаринов, Вестн. Моск. ун-та. Сер. матем., механ., 2003, № 3, 67-76. 
[16] Я.В. Татаринов, Уравнения классической механики в лаконичных формах, http://hal.ccsd.cnrs.fr/ccsd-00015612, Изд-во Центра прикладных исследований при мех.-мат. ф-те МГУ, М., 2005, 88 с.

[17] М. А. Приходько, Матем. заметки, 78:5 (2005), 727-744.

[18] М. А. Приходько, ТМФ, 148:3 (2006), 444-458.

[19] В.Н. Сорокин, Упругие столкновения бъстрых электронов с релятивистским атомом водорода, Препринт Института прикладной математики им. М. В. Келдыша РАН № $71,2006$.

[20] В. Н. Сорокин, О модели Козлова-Никишина, Препринт Института прикладной математики им. М. В. Келдыша РАН № 24, 2007.

[21] Б. А. Воронцов-Вельяминов, Астрономия. Учебник для 10 класса, Просвещение, М., 1976.

[22] С. Вейнберг, Гравитация и космология. Принципы и приложения общей теории относительности, Мир, М., 1975.

[23] Н. П. Коноплева, УФН, 123:4 (1977), 537-563.

[24] Н. Т. Роузвер, Перигелий Меркурия. От Леверъе до Эйнштейна, Мир, М., 1985.

[25] Р. А. Асанов, Г. Н. Афанасьев, ЭЧАЯ, 27:3 (1996), 713-746.

Поступила в редакцию 14.05.2010 